Annales de Parasitologie (Paris), t. 43, 1968, $\mathrm{n}^{\circ} 1$, pp. 79 à 85

\title{
Actions histopathologiques comparées de parasites coelomiques des larves de Simulies (Chytridiales, Microsporidies)
}

\author{
par Jean MAURAND et Jehanne-Françoise MANIER \\ (Equipe de Recherches de Protistologie associée au C.N.R.S. ( $\mathrm{P}^{\mathrm{r}}$ O. TUZET) \\ Faculté des Sciences F. 34 -Montpellier)
}

\begin{abstract}
Résumé
Nous pouvons classer provisoirement un certain nombre de parasites colomiques des larves de Simulies en trois groupes:

$1^{\circ}$ Coelomycidium simulii ne provoque pas de réaction de la part du tissu-hôte. Il se localise électivement dans le tissu adipeux et le tissu pigmentaire (accessoirement dans le tissu nerveux).

$2^{\circ}$ Thelohania bracteata, Plistophora simulii, P. debaiseuxi attaquent le tissu adipeux et induisent une légère hypertrophie et éosinophilie des noyaux, hypertrophient les cellules sanguines et épithélio-intestinales.

$3^{\circ}$ Stempellia simulii induit une intense réaction du tissu adipeux parasité : hypertrophies nucléaire et cytoplasmique.

Tous ces parasites sont mortels pour l'hôte.
\end{abstract}

\section{Summary}

At present some cælomic parasites in Simulium larvae can be put into three classes:

$1^{\circ}$ Coelomycidium simulii does not bring on reactions in the host-tissue. $C$. $s$. mainly attacks the adipose tissue and chromatocytes (sometimes the nervous system).

$2^{\circ}$ Thelohania bracteata, Plistophora simulii, P. debaiseuxi attack the adipose tissue and infer a slight hypertrophy and eosinophilic reaction of the nuclei and the blood cells and intestinal cells greatly increase.

$3^{\circ}$ Stempellia simulii brings on a deep reaction of the parasitized fat body: nuclear and cytoplasmic hypertrophy.

All these parasites are mortal for Simulium larvae. 
Tous les auteurs qui ont étudié les Microsporidres parasites des larves de Simulies ont signalé l'envahissement du corps adipeux larvaire. Mais, surtout préoccupés par l'étude du cycle de la Microsporidie, ils n'ont pas cherché à savoir si ces protistes avaient une spécificité tissulaire stricte et ils n'ont pas étudié la réaction d'une cellule ou d'un tissu à une attaque par les Microsporidies. L'un de nous (Maurand, 1967) a essayé de préciser les relations de Thelohania bracteata (Strickland, 1913) et de Plistophora simulii (Lutz et Splendore, 1904) avec différentes larves de Simulies en particulier, ce qui concerne les rapports tissus-parasite (1). Nous nous proposons de poursuivre ici ce premier travail par l'adjonction des effets histopathologiques de Coelomycidium simulii Debaisieux, 1919 (Phycomycètes, Chytridiales), Plistophora debaisieuxi Jirovec, 1943, et de Stempellia simulii Maurand-Manier, 1967. Nous estimons en effet qu'il est possible de comparer les effets histopathologiques de parasites aussi différents d'un point de vue systématique que les Chytridiales et les Microsporidies en raison du facteur commun que constitue le tissu généralement attaqué, le corps gras des larves de Simulies. Nous verrons, en outre, chemin faisant, que le corps gras de ces larves, très classiquement décrit comme étant le tissu parasité par ce parasite, n'est pas le seul mis en cause et que la question mérite d'être approfondie.

\section{COELOMYCIDIUM SIMULII.}

Une larve de Simulie infestée par Coelomycidium simulii se reconnaît à l'œil nu par la marbrure rouge vin de son corps. Des plasmodes parasitaires se développent à l'intérieur des cellules pigmentaires superficielles dont ils repoussent les granules colorés à la périphérie, ce qui a pour conséquence une concentration de la coloration et sa disposition grillagée. Lors de la résolution des plasmodes en leurs éléments flagellés, il y a dispersion des granules pigmentaires et la larve morte perd cette marbrure typique.

L'abdomen surtout, mais aussi le thorax sont bcurrés de plasmodes parasitaires. Le plasmode peut, en effet, se développer à l'intérieur d'une cellule sanguine qui le rend mobile passivement: emporté par le torrent circulatoire, on le retrouve jusque dans les prémandibules. Le tissu adipeux est cependant le milieu de choix pour le développement du parasite (Pl., fig. 6). Il y a augmentation de taille de la cellule parasitée sans qu'on puisse réellement parler d'hypertrophie, très rapidement disparition du noyau. Les plasmodes parasitaires ont la localisation des cellules adipeuses, sont fixes et nous ne mettons pas en évidence de réaction cellulaire à l'échelon microscopique ordinaire si ce n'est une légère distension de la membrane de la cellule-hôte. Celle-ci semble jouer un rôle de sac après qu'elle ait été vidée de son protoplasme; en fin d'évolution, le corps gras disparaît complètement.

Coelomycidium s'attaque aussi bien à l'adipeux interne qu'à l'adipeux périphérique (= chromatocytes). Il se loge même dans les cellules pigmentaires du testicule

(1) Seulement après la correction des épreuves de cet article, nous avons pu prendre connaissance d'un travail de Rubcov 1966, sur les rapports réciproques entre les larves de Simulies et les Microsporidies Plistophora simulii, P. debaisieuxi, Thelohania fibrata (Strickland, 1913), et T. varians (Léger, 1897). 
larvaire (Pl., fig. 5), du système nerveux. Enfin, nous l'avons localisé exceptionnellement à l'intérieur des ganglions cérébrö̈des; nous avons compté cinq kystes dans un même ganglion (Pl., fig. 4).

\section{L'Hôte et Les Microsporidies T. BRACTEATA, P. SIMULII, P. DEBAISIEUXI}

Nous ne reviendrons pas sur l'étude des rapports tissus des larves de SimuliesMicrosporidies: Thelohania bracteata (Strickland, 1913), Plistophora simulii (Lutz et Splendore, 1904), espèces auxquelles nous ajoutons Plistophora debaisieuxi Jirovec, 1943 pour son comportement similaire. Nous renvoyons en outre le lecteur à l'important travail de Rubcov, 1966. Ces Microsporidies sont essentiellement parasites du corps gras des larves, mais il est prouvé en outre, qu'elles peuvent être localisées dans les cellules sanguines, dans les cellules épithélio-intestinales qui prennent de ce fait une allure particulière avec gaine protoplasmique d'aspect fibreux et dans le complexe corps allate glande prothoracique. Les noyaux des cellules adipeuses sous influence parasitaire s'hypertrophient peu et deviennent éosinophiles tandis que les noyaux des cellules sanguines et intestinales croissent énormément.

\section{STEMPELLIA SIMULII.}

Stempellia simulii est parasite du corps adipeux chez lequel elle provoque d'importantes modifications (Pl., fig. 2). Elle semble spécifique de ce tissu et nous ne l'avons trouvée dans aucun autre. Elle rappelle par ses effets histopathologiques le parasitisme des cellules du canard par Leucocytozoon simondi Huff, 1942, ou bien celui de l'épinoche par Glugea anomala Weissenberg, 1921, deux parasites qui engendrent l'hypertrophie des cellules parasitées.

L'envahissement du tissu adipeux par le parasite se fait de proche en proche. Avant l'invasion, la ceilu'e adipeuse multiplie le nombre de ses globules protéiniques éosinophiles. Ensuite la réaction du tissu adipeux se traduit nettement par l'élaboration d'un nouveau protoplasma; les noyaux-hôtes s'hypertrophient et les membranes cellulaires disparaissent. La multiplication de globules protéiniques avant l'invasion, suggère l'existence d'un stimulus précédant le parasite. Kunkel, 1918, a décrit des processus de ce type chez Plasmodiophora brassicae.

Dans ce protoplasme néoformé, les stades végétatifs du parasite se multiplient et repoussent vers le centre de la tumeur sporontes, pansporoblastes et spores mûres ( $\mathrm{Pl}$., fig. 1). On peut donc dire grosso modo que la maturation est centripète. La structure d'une tumeur est la suivante :

$1^{\circ}$ une zone limitante externe résultant d'une condensation protoplasmique périphérique.

$2^{\circ}$ un tissu très développé, syncytial et d'aspect fibrillaire renfermant les stades parasitaires et les noyaux-hôtes hypertrophiés.

$3^{\circ}$ une masse de spores mûres au centre. 


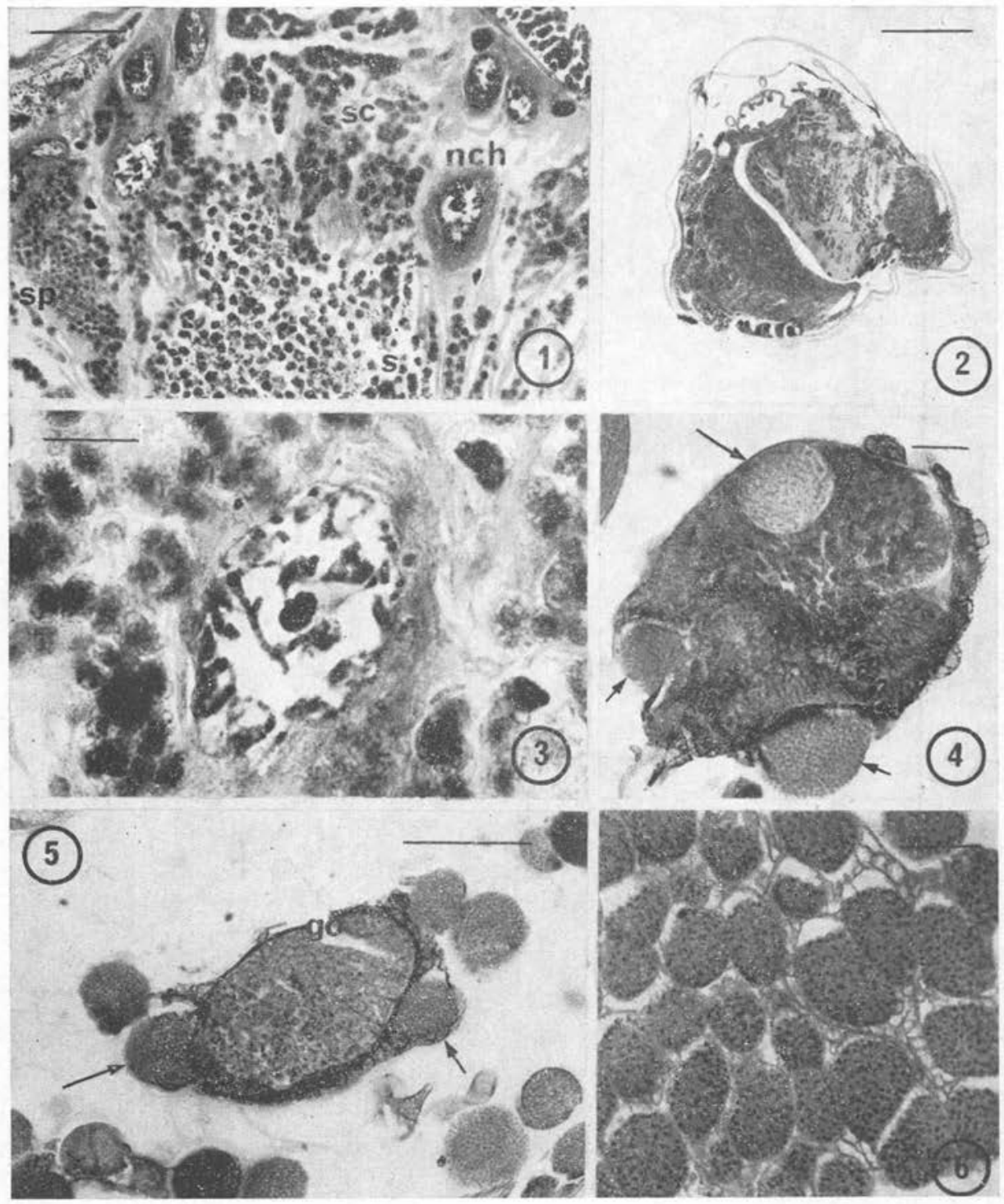

Planche 1. - Tumeur parasitaire de Stempellia simulii. nch: noyau de cellule-hôte; stades parasitaires jeunes: stades végétatifs (sc) et sporontes (sp); spores (s); (échelle $=30 \mu$ ).

Planche 2. - Vue d'ensemble d'une larve de Simulie parasitée par Stempellia simulii (coupe transversale); (échelle $=250 \mu)$

Planche 3. - Noyau de cellule-hôte hypertrophié sous l'action de Stempellia simulii ; (échelle $=10 \mu$ )

Planche 4. - Ganglion cérébroïde montrant trois plasmodes de Coelomycidium simulii (flèches); (échelle $=10 \mu)$

Planche 5. - Plasmodes de Coelomycidium simulii dans l'assise pigmentaire de la gonade (go); (échelle $=35 \mu)$

Planche 6. - Coelomycidium dans le tissu adipeux de la larve de Simulie; (échelle $=12 \mu$ ) 
Nous remarquons que le parasite induit d'abord une synthèse protoplasmique intense, mais qu'ensuite ce protoplasme disparaît progressivement au cours de l'évolution du parasite: les stades parasitaires jeunes (végétatifs et jeunes sporontes) sont entourés d'un protoplasma abondant, les pansporoblastes en cours de maturation, sont pris dans les mailles d'un filet protoplasmique, enfin les pansporoblastes renfermant les spores mûres sont en contact les uns avec les autres.

Nous avons donné (Maurand-Manier, 1967) les photographies prises au microscope électronique de ce tissu réactionnel comme élément de diagnose de l'espèce de Microsporidie. Le protoplasme montre une richesse particulière en mitochondries et en reticulum endoplasmique - d'où l'aspect fibrillaire en microscopie photonique - Ce tissu néoformé est bien de nature hôte puisque on ne connaît pas de mitochondries chez les Microsporidies, parasites intracellulaires stricts.

Les noyaux-hôtes augmentent considérablement de taille (de 2,5 $\mu$ à 30-40 $\mu$ ), des chromosomes géants apparaissent et rappellent ceux des glandes de la soie. Il y a certainement polyploïdie, le nucléole se divise (Pl., fig. 3). Ensuite, la chromatine devient pycnotique, on voit des figures amitotiques. En un stade ultérieur, la membrane se lyse et les nucléoles sont épars au milieu des spores mûres.

Ainsi l'énorme masse protoplasmique (noyaux hypertrophiés, cytoplasme riche en reticulum endoplasmique et mitochondries) de la tumeur jeune, est progressivement dissoute par le parasite. Une tumeur âgée constitue une mince enveloppe protoplasmique autour des spores mûres. En fin d'évolution, cette enveloppe cède sous la pression du parasite, les spores envahissent le cœlome et nous pouvons supposer que c'est à ce moment que survient la mort de l'hôte.

\section{SYNTHÈSE}

Après avoir récapitulé les tissus et organes dans lesquels nous avons mis les parasites en évidence, il sera intéressant d'analyser comparativement la réaction de plusieurs tissus à un parasite et inversement l'influence respective de plusieurs parasites sur un même tissu.

Le tableau ci-dessous indique les tissus et organes parasités: $P$. debaisieuxi, $P$. simulii, $T$. bracteata s'implantent dans des milieux variés, $S$. simulii semble inféodée au seul tissu adipeux (pigmentaire ou non), $C$. simulii est d'une exigence intermédiaire.

Le groupe de parasite G. I entraîne en général la disparition du protoplasme de toutes les cellules parasitées quelle que soit leur nature, une légère hypertrophie et perte de la basophilie des noyaux des cellules adipeuses, pigmentaires, endocrines. Par contre, les cellules sanguines et épithélio-intestinales parasitées augmentent sensiblemént de volume et leur noyau s'hypertrophie puis dégénère. Autour de ces cellules, il subsiste une gaine protoplasmique fibreuse anucléée.

Stempellia simulii (G. II), induit une réaction intense des cellules adipeuses et pigmentaires parasitées: réactivation des facultés synthétisantes de la cellule avec hypertrophie des noyaux et multiplication nucléolaire, cytoplasme abondant riche en ergastoplasme et mitochondries. 


\begin{tabular}{|c|c|c|c|c|c|c|c|c|c|c|}
\hline \multicolumn{2}{|c|}{ Parasite } & C.A. & C.P. & $\mathrm{H}$. & G.E. & E.I. & M. & T.M. & G.S. & S.N. \\
\hline G I & $\begin{array}{l}\text { P. deb. } \\
\text { P. sim. } \\
\text { T. bra. }\end{array}$ & + & + & + & + & + & - & 一 & - & - \\
\hline G II & S. sim. & + & + & - & - & - & - & - & - & - \\
\hline G III & C. sim. & + & + & + & - & - & - & - & - & + \\
\hline
\end{tabular}

Légende du tableau:
C.A. : corps adipeux
C.P. : cellules pigmentaires
H. : hémocytes
G.E. : glandes endocrines
E.I. : épithélium intestinal
M. : musculature
T.M. : tubes de Malpighi
G.S : glandes de la soie
S.N. : système nerveux
P. deb. : Plistophora debaisieuxi
P. sim. : P. simulii
T. bra. : Thelohania bracteata
S. sim. : Stempellia simulii
C. sim. : Coelomycidium simulii

Coelomycidium simulii (G. III) au contraire, « vide » la cellule adipeuse, pigmentaire ou sanguine de son cytoplasme et noyau. La membrane cellulaire est une enveloppe. Les plasmodes parasitaires dans les ganglions cérébroïdes sont rares et constituent un cas original de parasitisme.

Si maintenant nous analysons le comportement des tissus adipeux et pigmentaires vis-à-vis des trois groupes de parasites, nous notons trois types de comportement. Contre $S$. simulii, la cellule-hôte réagit violemment par hypertrophies cytoplasmique et nucléaire pour englober le parasite. Avec les trois autres Microsporidies, le cytoplasme disparaît et les noyaux s'hypertrophient légèrement. Enfin, lors d'un parasitisme à Coelomycidium, tout se passe comme s'il y avait dissolution pure et simple des cytoplasme et noyau, les membranes cellulaires seules subsistant.

En ce qui concerne les cellules sanguines, pour Coelomycidium, il y a à nouveau dissolution du contenu cellulaire, mais ici, les parasites du Groupe G. I, comme dans le cas de la cellule épithélio-intestinale, entraînent une hypertrophie cellulaire, dégénérescence du noyau, et la formation d'une gaine fibreuse.

En résumé, nous dirons qu'il y a deux parasites constants dans le choix du tissu et et dans leurs effets sur ce tissu: Stempellia simulii et Coelomycidium simulii. Par contre, les autres Microsporidies se montrent assez irrégulières quant à leur localisation et influence. 


\section{Bibliographie}

Debaisieux (P.), 1920. - Coelomycidium simulii n. g., n. sp. et remarques sur l'Amoebidium des larves de Simulies. La Cellule, 30, 244-277.

HufF (G. G.), 1942. - Schizogony and gametocyte development in Leucocytozoon simondi and comparison with Plasmodium and Hemoproteus. J. Insect. Dis., 71, 18-32.

JIROVEC (O.), 1943. - Revision der in Simulium larven parasitierenden Mikrosporidien. Zool. Anz., 142, 173-179.

Kunkel (O.), 1918. - Tissue invasion by Plasmodiophora brassicae. J. Agric. Research., 14, 543-572.

MAURAND (J.), 1967. - Relations Microsporidies-larves de Simulium: spécificité parasitaire d'hôte; spécificité parasitaire tissulaire. Ann. Par. Hum. Comp., 42, n 3, 285-290.

Maurand (J.), Manier (J.-F.), 1967. - Une Microsporidie nouvelle pour les larves de Simulies. Protistologica, sous-presse.

RuBCov (I. A.), 1966. - Rapports réciproques entre l'hôte et le parasite. Réponse des Simuliidae aux Microsporidies. Zh. Obshch. Biol. SSSR, 27, $\mathrm{n}^{\circ}$ 6, 647-661.

WeIssenberg (R.), 1921. - Zur Wirtsgewebsableitung der Plasmakörpers der Glugea anomala, Cysten. Arch. Protisten., 42, 400-21. 